\title{
9p21 index as estimated by dual-color fluorescence in situ hybridization is useful to predict urothelial carcinoma recurrence in bladder washing cytology
}

${ }^{\#}$ Shigeto Kawauchi ${ }^{1}$, ${ }^{\#}$ Hajime Sakai ${ }^{1,2}$, Kenzo Ikemoto ${ }^{1}$, Satoshi Eguchi ${ }^{1,3}$, Motonao Nakao ${ }^{1}$, Hirofumi Takihara ${ }^{4}$, Tomoyuki Shimabukuro ${ }^{5}$, Tomoko Furuya ${ }^{1}$, Atsunori Oga ${ }^{1}$,

Hideyasu Matsuyama ${ }^{3}$, Mutsuo Takahashi ${ }^{2}$, and Kohsuke Sasaki ${ }^{1}$

\#: The authors contributed equally to the present study.

Departments of Pathology ${ }^{1}$, Health Sciences ${ }^{2}$, and Urology ${ }^{3}$, Yamaguchi University Graduate School of Medicine, Yamaguchi, Japan

Department of Urology ${ }^{4}$, Sanyo-Onoda Municipal Hospital, Yamaguchi, Japan Department of Urology ${ }^{5}$, Ube Industry Central Hospital, Yamaguchi, Japan

Correspondence, galley, and reprint request to:

Shigeto Kawauchi, MD, PhD

Department of Pathology, Yamaguchi University Graduate School of Medicine, 1-1-1 Minamikogushi, Ube, Yamaguchi 755-8505, Japan

tel: +81 (836) 22-2222, fax: $+81(836) 22-2223$, e-mail: shig@yamaguchi-u.ac.jp 


\begin{abstract}
Recent studies have shown that chromosome 9p21 locus is frequently deleted in the early stages of urothelial carcinogenesis. To study the predictive value of the $9 \mathrm{p} 21$ aberrations in b-UC recurrence, dual-color fluorescence in situ hybridization (FISH) for 9p21 and chromosome 9 centeromere was applied to the bladder washing cytology (BWC) samples that were obtained from the b-UC patients treated by transurethral resection (TUR). For the evaluation, the 9p21 index (9p21-I) was defined as the ratio of the mean number of $9 \mathrm{p} 21$ signals per nucleus for that of the chromosome 9 centromere signals per nucleus in each of the BWC samples. The 9p21-I values of the BWC samples with no (G0) cytological atypia were significantly higher than those of the BWC samples with moderate $(\mathrm{G} 2)(P<0.01)$ and severe $(\mathrm{G} 3)(P<0.001)$ cytological atypia, but did not statistically differ from those of the BWC samples with mild (G1) cytological atypia. Recurrence free survival in the patients with a low 9p21-I value $(<0.9)$ was significantly poorer in comparison to the patients with a high 9p21-I value $(>0.9)$. Furthermore, two patients of BWC G1 with a low 9p21-I value recurred much sooner than the other patients of the BWC G1 category. These findings indicate that a decreased 9p21-I value is associated with b-UC recurrence and the 9p21-I may be useful as a marker to identify patients with elevated risk of b-UC recurrence.
\end{abstract}


Abbreviations: bladder washing cytology (BWC); fluorescence in situ hybridization (FISH); 9p21 index (9p21-I); mean 9p21 signal number per nucleus (MN-9p21); mean chromosome 9 centromere signal number per nucleus (MN-CH9); transurethral resection (TUR); urothelial carcinoma of the urinary bladder (b-UC)

Keywords: bladder washing cytology; fluorescence in situ hybridization; 9p21 index; recurrence; urinary bladder; urothelial carcinoma

Duality of interest: None declared 


\section{Introduction}

Urothelial carcinoma of the urinary bladder (b-UC) constitutes more than $80 \%$ of all bladder cancers [1]. In general, newly diagnosed superficial b-UCs consist of approximately $70 \%$ of noninvasive tumors and $30 \%$ of superficially invasive tumors. The patients with a noninvasive or minimally invasive b-UC show a favorable prognosis. Most of these patients are treated by transurethral resection (TUR), followed by periodic negative follow-up cystoscopy and bladder washing cytology (BWC) [2]. These tumors sometimes recur locally and grow superficially without marked muscular invasion, however, a small proportion of the tumors, approximately 5-10\%, progresses to invasive cancer [3]. The indicators of an increased recurrence risk include high tumor stage and grade, multifocality, and the presence of carcinoma in situ [2, 3].

Although cystoscopy is an effective method for the detection of b-UC, the method is essentially invasive and thus causes discomfort to the patients. Furthermore, early b-UC and carcinoma in situ may be difficult to detect solely by cystoscopy. On the other hand, cytology is easy to perform and is also non-invasive for the patients. Although cytology reveals high specificity in the detection of b-UC, the sensitivity is not always satisfactory and it may be even poor in low-grade b-UC $[4,5]$. One of the reasons for the unsatisfactory sensitivity can be explained by the fact that occasionally the bland-looking appearance of low-grade b-UC cells makes it difficult to distinguish them from the hyperplastic or the degenerated, non-neoplastic urothelial cells. Therefore, some ancillary techniques that can improve the diagnostic accuracy of cytology are believed to benefit the early detection of recurrent b-UC and the improved prognosis of the patients.

Recent studies have shown that superficial papillary b-UCs and the invasive nodular ones are unique in the genetic pathway [6-8]. In superficial papillary b-UCs, the most common genetic 
alterations are loss of heterozygosity ( $\mathrm{LOH})$, deletion of chromosome 9, and the activating-type mutations of the fibroblast growth factor receptor 3 (FGFR3) gene. However, invasive nodular b-UCs are characterized by the p53 tumor suppressor gene mutations and by chromosomal instability. Considering these genetic aberrations that are unique to b-UCs, it seems rational to apply some molecular method as a diagnostic aid of b-UC to cytology.

UroVysion $^{\mathrm{TM}}$ test has recently been developed [9] and used as an aid in cytology for urothelial cancer $[10,11]$. The test examines numerical aberrations of chromosomes $3,7,17$, and the 9p21 locus by using a multicolor fluorescence in situ hybidization (FISH) technique. Because the b-UC cells have rather unique genetic alterations as described above, the UroVysion ${ }^{\mathrm{TM}}$ test is useful to distinguish the b-UC cells from the diagnostically problematic, non-neoplastic urothelial cells. In our recent study using surgically resected b-UC tissues, the UroVysion ${ }^{\mathrm{TM}}$ test revealed much more frequent numerical aberrations in the 9p21 locus in comparison to the other chromosomes [12], and a similar finding was also reported by others [10]. Furthermore, the 9p21 numerical aberration was observed not only in superficial papillary b-UCs, but laso in invasive nodular b-UCs. The high incidence of the $9 \mathrm{p} 21$ aberration in b-UCs prompted us to investigate the utility of $9 \mathrm{p} 21$ in differentiating the diagnostically problematic b-UC cells in BWC.

In the present study, we devised the $9 \mathrm{p} 21$ index (9p21-I) to assess the relative extent of 9p21 DNA copy number alterations in the bladder washing cytology (BWC) samples by using the dual-color FISH method. The aim of the present study was to investigate the significance of 9p21 aberrations, especially 9p21 loss, in BWC samples as a marker to predict cancer recurrence in the patients with b-UC that were treated by TUR. 


\section{Materials and methods}

\subsection{Materials}

The BWC samples were obtained from sixty-one patients at the time of negative follow-up cystoscopy in the Sanyo-Onoda Municipal Hospital, the Ube Industry Central Hospital, and the Yamaguchi University Hospital. All the patients had a past-history of b-UC and were initially treated by TUR. The histopathologic diagnoses and grading were reviewed based on the World Health Organization Classification, 2004 [1]. The stages of the primary b-UCs were classified according to the TNM Classification of Carcinomas of the Urinary Bladder [1]. With respect to the recurrence-free survival of the patients, a b-UC recurrence was considered present when a urinary bladder biopsy confirmed recurrent b-UC. The present study followed the ethical guidelines of the Institutional Review Board of Yamaguchi University Graduate School of Medicine.

\subsection{Bladder washing cytology}

The bladder washings were obtained by instillation of $100 \mathrm{ml}$ of $0.9 \%$ sodium chloride solution into the urinary bladder after a follow-up cystoscopy. An equal volume of $50 \%$ ethanol was immediately added to the samples to preserve the cells before the start of the cytological examination. The bladder washings were centrifuged at $1400 \mathrm{rpm}$ for 10 minutes using a centrifuge (05PR-22, Hitachi, Tokyo, Japan) and the pellets were resuspended into $10 \mathrm{ml}$ of supernatant and recentrifuged. The sediments were centrifuged with an autosmear centrifuge (CF-120, Sakura, Tokyo, Japan), and were spread over two or three silane-coated glass slides. The smear slides were immediately fixed with $95 \%$ ethanol and one of the prepared slides was stained by the standard Papanicolaou method. 
The cytological estimation for the BWC slides was done according to the previously described set of criteria [13]: cytological grade $0(\mathrm{G} 0)=$ no atypia, cytological grade $1(\mathrm{G} 1)=$ mildly atypia (cannot rule out low-grade bladder cancer), cytological grade $2(\mathrm{G} 2)=$ moderate atypia (suspicious of bladder cancer), and cytological grade $3(\mathrm{G} 3)$ = severe atypia (diagnostic of high-grade bladder cancer or carcinoma in situ).

\subsection{Dual-color fluorescence in situ hybridization}

The bladder washing smear slides were incubated with $0.05 \%$ pepsin at $37^{\circ} \mathrm{C}$ for 10 minutes and washed twice with phosphate-buffered saline, $\mathrm{pH} 7.4$, (PBS) at room temperature (RT) for 5 minutes. The slides were then fixed in $1 \%$ formaldehyde solution for 3 minutes, washed twice in PBS at RT for 5 minutes, re-fixed in Carnoy's solution for 5 minutes, dehydrated in each 70\%, 85\% and $100 \%$ ethanol solution for 2 minutes, and air-dried. The slides were denatured in $70 \%$ formamide / $2 \mathrm{x}$ standard sodium citrate $(2 \mathrm{xSSC})$ at $73^{\circ} \mathrm{C}$ for 5 minutes, dehydrated in a series of $70 \%, 85 \%$ and $100 \%$ ethanol solutions for each 2 minutes and air-dried. The dual-color FISH probe mixture for the chromosome 9p21 locus and the chromosome 9 pericentromeric region (Figure 1a) (LSI-9p21 Spectrum-Orange / CEP-9 Spectrum-Green Probe, Abbott-Vysis, Downers Grove, IL, USA) was denatured for 5 minutes in a water bath at $73^{\circ} \mathrm{C}$ and applied to the denatured sample slides. The slides were covered with cover glasses, sealed with rubber cement, and incubated at $37^{\circ} \mathrm{C}$ for 72 hours in a humidified chamber. Post-hybridization washes were made in $0.4 \mathrm{x}$ SSC / $0.3 \% \mathrm{NP}-40$ for 2 minutes at $73^{\circ} \mathrm{C}$ and $2 \mathrm{x}$ SSC / 0.1\% NP-40 at room temperature for 2 minutes twice, followed by air-drying and counterstaining with a DAPI-II antifade solution (Vysis). 


\subsection{Enumeration of fluorescence in situ hybridization signals}

The hybridized BWC slides were scored for FISH signals on each urothelial cell nucleus using a BX60 fluorescence microscope (Olympus, Tokyo, Japan). In the enumeration of FISH signals, overlapping cells and cells with indistinct and blurry signals were not scored. The numbers of the 9p21 signals and the chromosome 9 centromere signals were separately counted in each urothelial cell nucleus and the mean signal numbers were calculated per 100 urothelial cells in each BWC slide. The 9p21 index (9p21-I) was defined as the ratio of the mean 9p21 signal number per nucleus (MN-9p21) for the mean chromosome 9 centromere signal number per nucleus (MN-CH9) in each BWC sample FISH slide.

\subsection{Statistical analysis}

The one-way factorial analysis of variance and multiple comparisons (Turkey-Kramer test) were used to estimate the numerical difference in the MN-9p21, MN-CH9, and the 9p21-I values categorized by the BWC grades. The figures are described as mean \pm standard deviation. The recurrence-free survival was compared between the patients with high and low 9p21-I values using the Kaplan-Meier method and the log-rank test. A two-tailed $P<0.05$ was considered significant. 


\section{Results}

\subsection{Clinicopathologic findings, bladder washing cytology, and dual-color fluorescence in situ hybridization}

The age of the patients ranged from 50 to 95 years (mean, 73.1 years) and the stages of the primary b-UCs were 33 pTa, 24 pT1, 2 pT2, and 2 tumors without any information regarding stage. The other clinicopathologic characteristics are summarized in Table 1. The 61 BWC specimens were classified into 16 samples of the G0 category, 22 of G1, 13 of G2, and 10 of the G3 category by the conventional cytological examination based on the Papanicolaou stain (Figures 1b, 1d, 1f and Table 1). In most of the FISH sample slides, a significant number of informative urothelial cells were observed with distinct fluorescence signals (Figures 1c, 1e, 1g). The percentages of the informative urothelial cells per total urothelial cells observed were $85 \%$ to $97 \%$ in the BWC G0 category, $70 \%$ to $95 \%$ in the G1, $75 \%$ to $87 \%$ in the G2, and $71 \%$ to $85 \%$ in the BWC G3 category. Furthermore, it was not so dificult to differentiate the cancerous urothelial cells from the inflammatory and fibroblastic stromal cells even under the fluorescence microscopy as described previously [9].

\subsection{9p21 index, cytological grade and clinicopathologic features}

The 9p21-I values of all the BWC samples ranged from 0.759 to $1.021(0.942 \pm 0.058)$. The MN-9p21 of all the BWC samples ranged from 1.81 to $2.16(2.00 \pm 0.089)$. The MN-CH9 of all the BWC samples ranged from 1.98 to $2.50(2.14 \pm 0.161)$. The distributions of the 9p21-I, MN-9p21, and MN-CH9 values as classified by the BWC grade are demonstrated in Table 2 and Figures 2a, $2 b, 2 c$. 
Statistically, the 9p21-I values of the BWC G0 samples were significantly higher in comparison to those of the BWC G2 $(P<0.01)$ and the BWC G3 $(P<0.001)$ samples (Figure 2a). The 9p21-I values of the BWC G1 samples were significantly higher in comparison to those of the BWC G2 $(P$ $<0.01)$ and the G3 $(P<0.01)$ samples. There were no significant differences in 9p21-I between the BWC G0 and the BWC G1 samples, thus indicating that these BWC G0 and G1 samples could be included in a category from the viewpoint of 9p21-I. Furthermore, there were no significant differences in 9p21-I between the BWC G2 $(P<0.01)$ and the G3 $(P<0.01)$ samples, indicating that the BWC G2 and the G3 samples could be included in a category from the viewpoint of 9p21-I. As for the MN-9p21 (Figure 2b) and the MN-CH9 (Figure 2c), there was no such a statistical significance as 9p21-I when the BWC samples were categorized by the cytological grade.

\subsection{9p21 index and recurrence}

The median follow-up period of the patients was 22.8 months (range, 2 months to 114 months). The patients of the BWC G3 category tended to have an elevated risk of recurrence in comparison to those of the other categories, but statistically insignificant. The recurrence-free survival of the patients with a low 9p21-I value $(<0.9)$ was significantly poorer in comparison to those with a high 9p21-I value (>0.9) (Figure 3).

Two patients from the BWC G1 category that revealed significantly lower 9p21 index values, 0.759 and 0.843 , both of which were below the 5 th percentile in the $9 \mathrm{p} 21-\mathrm{I}$ values of the BWC G1 category, shortly developed BWC G3 and recurrent UC within 2 months after the completion of the present study (Figure 2a, small arrows). 


\section{Discussion}

Recent studies have shown that the chromosome 9p21 locus is one of the most frequent genetic aberrations in b-UCs $[14,15]$. It is well known that the 9p21 locus contains the INK4A and the ARF genes encoding the tumor suppressor proteins that suppress the G1-S transition of the cell cycle, p16 and p19, respectively [16]. Homozygous and hemizygous deletions of the INK4A gene are one of the most common alterations in b-UCs and occur early in the development of papillary b-UC as well as in b-UC in situ $[16,17]$.

The INK4A gene product, p16, forms complexes with cyclin-dependent kinases (CDKs)-4 and -6, and inhibits phosphorylation of the $\mathrm{pRB}$ tumor suppressor protein, and therefore inactivates the E2F transcription factor that promotes the G1-S transition of the cell cycle (pRB pathway) [18]. Furthermore, the INK4B gene product p19 interacts with the MDM2 protein, suppresses MDM2-induced degeneration of the p53 protein, and as a result, promotes apoptosis and G1 arrest of the cell cycle (p53 pathway) [18]. Abnormalities in the pRB and p53 pathways have been shown to be prevalent in many human cancers. Because an autonomic cell proliferation is one of the most fundamental characteristics of cancer, frequent abnormalities in these pathways seem to explain an intimate association between the 9p21 alterations, especially 9p21 loss, and the elevated cell proliferation in a variety of human cancers including b-UC.

In BWC examinations, atypical-looking urothelial cells seem to be classified into two categories, i.e., degenerated or hyperplastic non-neoplastic urothelial cells and dysplastic or cancerous urothelial cells [19]. These cells may sometimes be difficult to differentiate from each other by conventional morphology-based examinations. Considering that $9 \mathrm{p} 21$ is frequently deleted even in the early stages of urothelial carcinogenesis [20] and that the non-neoplastic urothelial cells carry 
rare or no chromosomal aberrations, especially in chromosome 9 [21], atypical urothelial cells with numerical aberrations of $9 \mathrm{p} 21$ locus are much more likely to be neoplastic. Taking this into consideration, we investigated the significance of the 9p21 aberration in the BWC samples to detect early recurrence of b-UC.

However, for example, if an urothelial cell with simultaneous, two 9p21 signals and three or more chromosome 9 centromere signals are present, such a relative 9p21 loss cannot be recognized by a conventional single-color FISH examination for the 9p21 locus. Taking these situations into consideration, we have devised the 9p21-I to objectively assess the 9p21 imbalances. Because the $9 \mathrm{p} 21-\mathrm{I}$ is defined as the ratio of the average number of $9 \mathrm{p} 21$ signals for that of chromosome 9 centromere signals in each BWC sample FISH slide, the index is expected to estimate the 9p21 alterations more quantitatively. Furthermore, the cytological grade of the b-UC samples demonstrated a statistically significant correlation with the 9p21-I, but not with the average number of 9 p21 signals. These findings indicated the utility of the 9p21-I as a marker to differentiate precancerous (probably "dysplatic") or cancerous urothelial cells from suspicious ones with so-called benign atypia.

In the present study, the dual-color FISH examination detected a number of urothelial cells with 9p21 loss even in the BWC G0 samples. Although a small proportion of the urothelial cells with a reduced 9p21 signal number might be those with artificially damaged 9p21 loci, most of these urothelial cells were rather considered to carry the true cytogenetical aberration, i.e., 9p21 deletion and to be at least potentially cancerous in character. Therefore, the $9 \mathrm{p} 21$ loss as estimated by a reduced 9p21-I value may be used as a marker to screen patients with elevated risk of b-UC recurrence. In the present study, recurrence free survival in the patients with a low 9p21-I value $(<$ 
0.9) was significantly poorer in comparison to the patients with a high 9p21-I value $(>0.9)$. Furthermore, two patients of BWC G1 with a low 9p21-I value recurred much sooner than the other patients of the BWC G1 category. These findings are thought to support our idea that a decreased 9p21-I value is associated with b-UC recurrence.

It is difficult to fully explain the urothelial carcinogenesis in the present FISH analysis. In many human cancers, INK4A and ARF are inactivated mainly by allelic deletions [20, 22], silencing mutations [20, 23], and/or promoter 5'-CpG island methylation [24]. Because silencing or loss-of-function mutations of INK4A and ARF genes are relatively rare in b-UCs [16, 17, 20], allelic deletion and promoter $\mathrm{CpG}$ methylation may be major silencers for these genes. Therefore, the analysis regarding the INK4A and ARF promoter methylation [25] in combination with the 9p21 deletion may be more useful for detecting recurrent b-UC using BWC samples.

In conclusion, we have devised the 9p21-I to quantitatively assess the extent of 9p21 deletion by the dual-color FISH method for BWC samples. A reduced 9p21-I value is significantly associated with the elevated risk of b-UC recurrence. The 9p21-I may thus be useful as a tool to screen post-TUR patients with elevated risk of b-UC recurrence. 


\section{References}

[1] Lopez-Beltran A, Knowles MA, Sauter G, et al. Infiltrating urothelial carcinoma. In: Eble JN, Sauter G, Epstein JI, SesterhennIA, editors. World Health Organization classification of tumors: Pathology and genetics of tumors of the urinary system and male genital organs. 1st ed. Lyon, France: IARC Press; 2004. p. 90-109.

[2] Donat SM. Evaluation and follow-up strategies for superficial bladder cancer. Urol Clin North Am 2003; 30: 765-76.

[3] Kiemeney LA, Witjes JA, Heijbroek RP, Verbeek AL, Debruyne FM. Predictability of recurrent and progressive disease in individual patients with primary superficial bladder cancer. J Urol 1999; 150: 60-4.

[4] Bastacky S, Ibrahim S, Wilczynski SP, Murphy WN. The accuracy of urine cytology in daily practice. Cancer 1999; 87: 118-28.

[5] Ramakumar S, Bhuiyan J, Besse JA, et al. Comparison of screening methods in the detection bladder cancer. J Urol 1999; 161: 388-94.

[6] Spruck CH, Ohneseit PF, Gonzalez-Zulueta M, et al. Two molecular pathways to transitional cell carcinoma of the bladder. Cancer Res 1994; 54: 784-8.

[7] Wu XR. Urothelial tumorigenesis: A tale of divergent pathways. Nat Rev Cancer 2005; 5: 713-25.

[8] Woff EM, Liang G, Jones PA. Mechanisms of disease: Genetic and epigenetic alterations that drive bladder cancer. Nat Clin Pract Urol 2005; 2: 502-10.

[9] Sokolova IA, Halling KC, Jenkins RB, et al. The development of a multitarget, multicolor fluorescence in situ hybridization assay for the detection of urothelial carcinoma in urine. $\mathrm{J}$ 
Mol Diagn 2000; 2: 116-23.

[10] Zellweger T, Benz G, Cathomas G, et al. Multi-target fluorescence in situ hybridization in bladder washings for prediction of recurrent bladder cancer. Int J Cancer 2006; 119: 1660-5.

[11] Skacel M, Fahmy M, Brainard JA, et al. Multitarget fluorescence in situ hybridization assay detects transitional cell carcinoma in the majority of patients with bladder cancer and atypical or negative urine cytology. J Urol 2003; 169: 2101-5.

[12] Ikemoto K, Furuya T, Matsuda K, et al. Multicolor FISH and cytometric analyses allow classification of urothelial carcinomas into two subtypes, low- and high-grade tumors. Int $\mathbf{J}$ Oncol 2004; 25: 893-8.

[13] Esposti PL, Zajicek J. Grading of transitional cell neoplasms of the urinary bladder from smears of bladder washings. A critical review of 326 tumors. Acta Cytol 1972; 16: 529-37.

[14] Cairns P, Shaw ME, Knowles MA. Initiation of bladder cancer may involve deletion of a tumor-suppressor gene on chromosome 9. Oncogene 1993; 8: 1053-5.

[15] Ruppert JM, Tokino K, Sidransky D. Evidence for two bladder cancer suppressor loci on human chromosome 9. Cancer Res 1993; 53: 5053-5.

[16] Orlow I, Lacombe L, Hannon GJ, et al. Deletion of p16 and p15 genes in human bladder tumors. J Natl Cancer Invest 1995; 87: 1524-9.

[17] Orlow I, LaRue H, Osman I, et al. Deletions in the INK4A gene in superficial bladder tumors: Association with recurrence. Am J Pathol 1999; 155: 105-3.

[18] Sherr CJ. The Pezcoller lecture: cancer cell cycles revised. Cancer Res 2000; 60: 3689-95. 
[19] Koss LG. Cytology of tumors of the bladder. In: Koss LG, Melamed MR, editors. Koss' diagnostic cytology and its histopathologic bases. 5th ed. Philadelphia, PA: Lippincott Williams \& Wilkins; 2006. p. 791-09.

[20] Czerniak B, Chaturvedi V, Li L, et al. Superimposed histologic and genetic mapping of chromocsome 9 in progression of human urinary bladder neoplasia: Implications for a genetic model of multistep urothelial carcinogenesis and early detection of urinary bladder cancer. Oncogene 1999; 18: 1185-95.

[21] Yin M, Bastacky S, Parwani AV, McHale T, Dhir R. p16 $6^{\text {ink4 }}$ immunoreactivity is a reliable marker for urothelial carcinoma in situ. HUM PATHOL 2008; 39: 527-35.

[22] Zhang Y, Xiong Y, Yarbrough WG. ARF promotes MDM2 degradation and stabilizes p53: ARF-INK4a locus deletion impairs both the Rb and p53 tumor suppression pathways. Cell 1998; 92: 725-34.

[23] Hussussian CJ, Struewing JP, Goldstein AM, et al. Germline p16 mutations in familial melanoma. Nat Genet 1994; 8: 15-21.

[24] Merio A, Herman JG, Mao L, et al. 5' $\mathrm{CpG}$ island methylation is associated with transcriptional silencing of the tumor suppressor p16/CDKN2/MTS1 in human cancers. Nat Med 1995; 1: 686-92.

[25] Dulaimi E, Uzzo RG, Greenberg RE, Al-Saleen T, Cairns P. Detection of bladder cancer in urine by a tumor suppressor gene hypermethylation panel. Clin Cancer Res 2004; 10: 1887-93. 


\section{Figure Legends}

Figure 1 (a) Chromosome 9 ideogram. The 9p21 and chromosome 9 centromere regions are indicated as red and green bold lines, which are recognized by SpectrumRed-labeled and SpectrumGreen-labeled fluorescence in situ hybridization (FISH) probes, respectively. (b, d, f) Representative urothelial cells in bladder washing cytology (BWC). The urothelial cells that are observed in the BWC specimens and are classified as grade 0 (b), 1 (d), and 3 (f) (x 325, modified Papanicolaou stain). (c, e, g) Representative urothelial cells in dual-color fluorescence in situ hybridization (FISH) with 9p21 locus-specific and chromosome 9 centromere probes. The FISH figures $\mathbf{c}, \mathbf{e}$, and $\mathbf{g}$ correspond to the BWC samples $\mathbf{b}$, $\mathbf{d}$, and $\mathbf{f}$, respectively. The red signal indicates a 9p21 region and the green signal represents a chromosome 9 centromere region. In a BWC grade 0 sample, two 9p21 signals and two chromosome 9 centromere signals are observed in the nucleus (c, original magnification x 400). In a BWC grade 1 sample, one 9p21 signal and two chromosome 9 centromere signals are observed in each nucleus (e, original magnification $\times 375$ ). In a BWC grade 3 sample, two 9p21 signals and two to five chromosome 9 centromere signals are observed in the nuclei (g, original magnification x 375).

Figure 2 (a, b, c) Correlations of cytological grade in bladder washing cytology (BWC) with 9p21 index (9p21-I)(a), mean number of 9p21 locus per nucleus (MN-9p21) (b), and mean number of chromosome 9 centromere per nucleus (MN-CH9) (c). (a) The 9p21-I was defined as the ratio of the MN-9p21 for the MN-CH9 in each of the BWC samples. 
Upper figures demonstrate statistical probability in the differences of the 9p21-I among the BWC samples categorized by the cytological grade. Small arrows indicate two patients that developed recurrent urothelial carcinoma sooner than the other patients within the BWC G1 category. A boxplot analysis reveals that both are outliers of the 9p21-I in the BWC G1 category. (b, c). A statistical difference is not evident in the MN-9p21 (b) and the MN-CH9 (c) when the BWC samples were categorized by the cytological grade.

Figure 3 Recurrence-free survival curves stratified for low and high 9p21 index in FISH for bladder washing cytology samples. A low 9p21-I value $(<0.9)$ is significantly associated with poor recurrence-free survival $(P<0.05)$. 
Figure(s)
Click here to download high resolution image

Figure 1

a
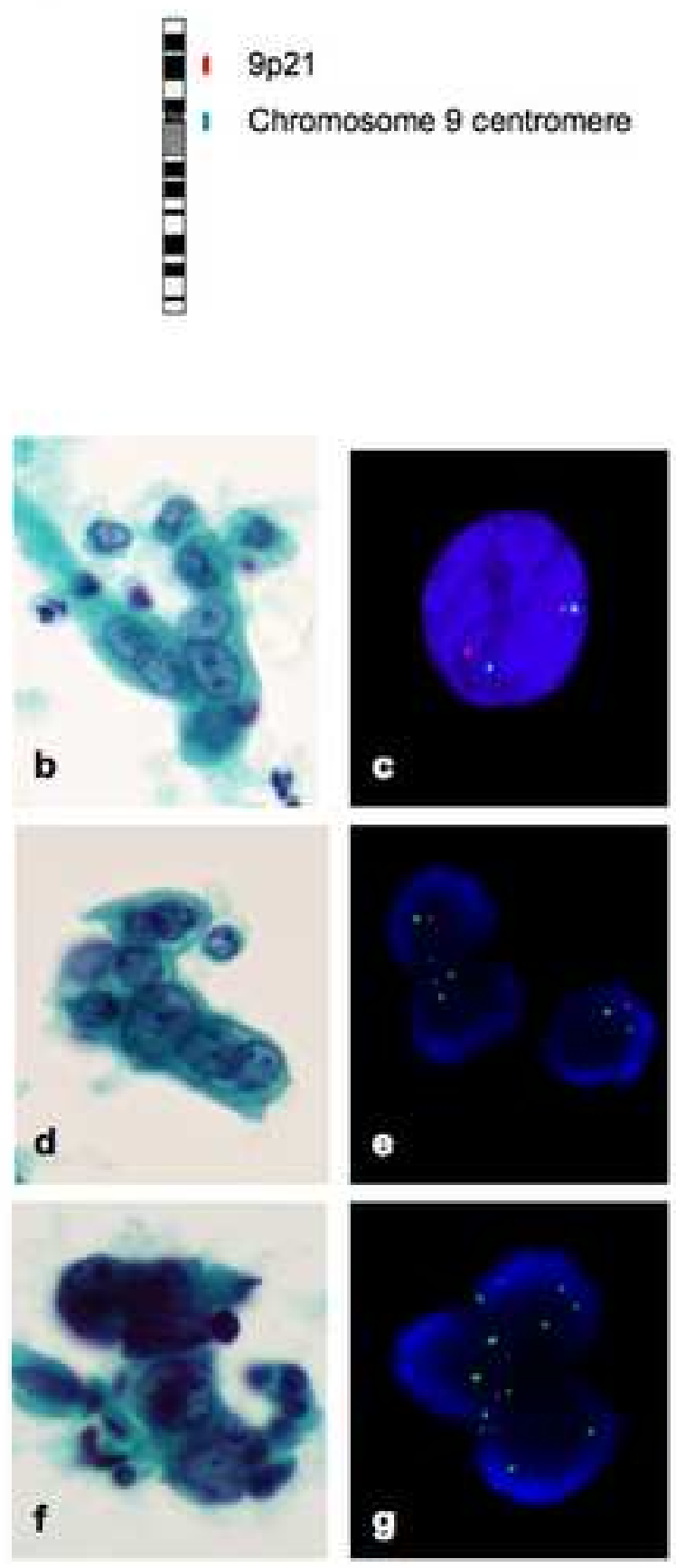
Click here to download high resolution image

Figure 2

a.

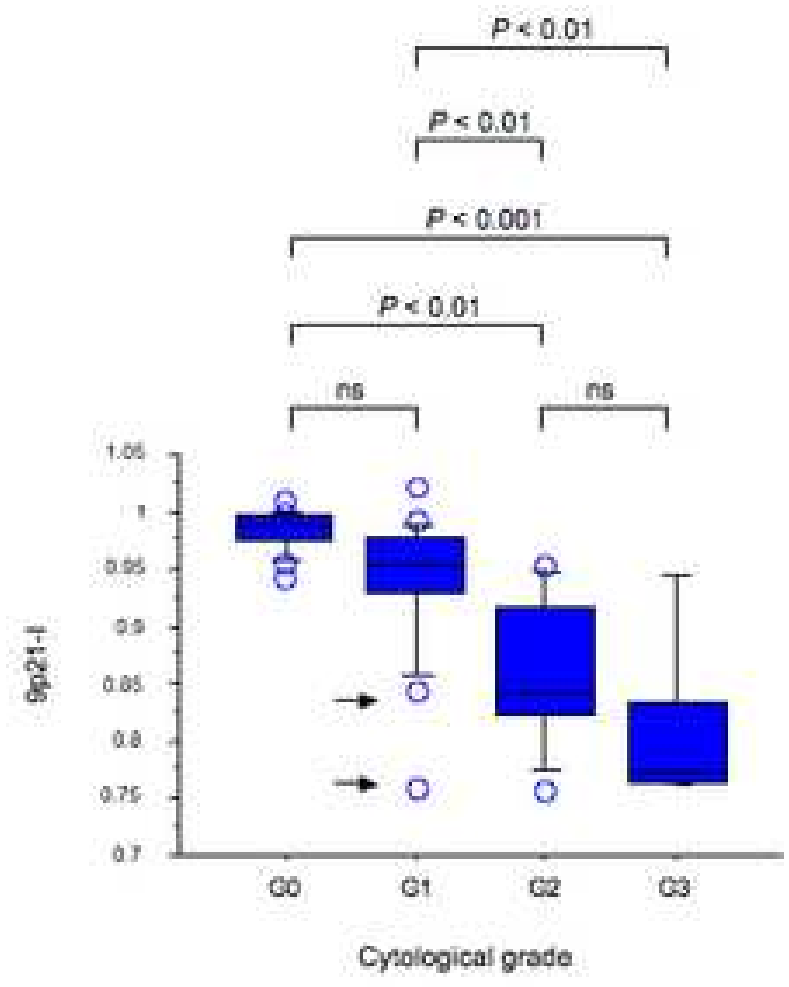

(continued) 
Click here to download high resolution image

Figure 2 (continued)

b.

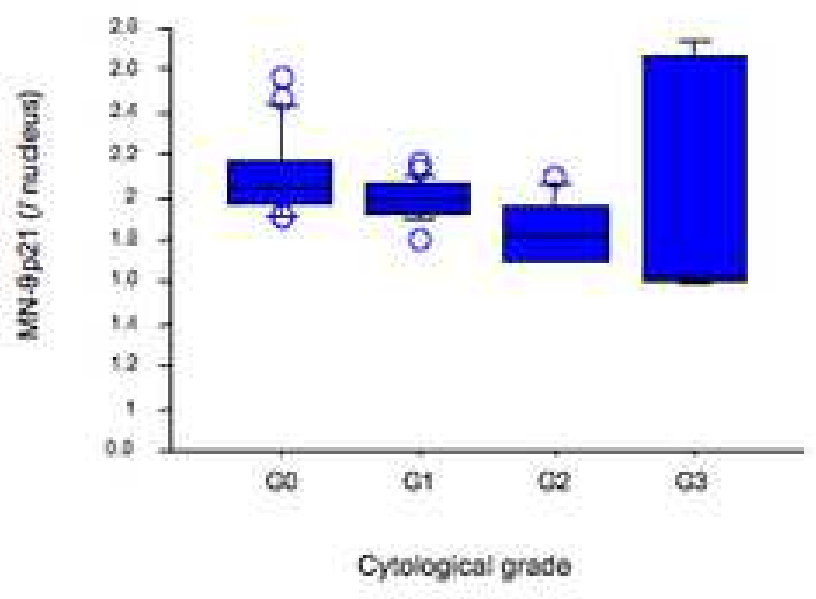

c.

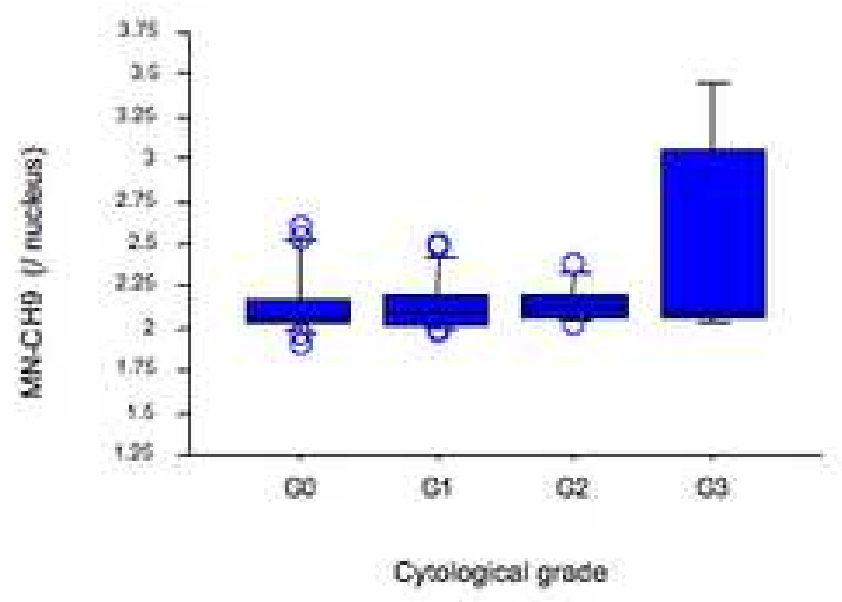




\section{Figure(s)}

Click here to download high resolution image

\section{Figure 3}

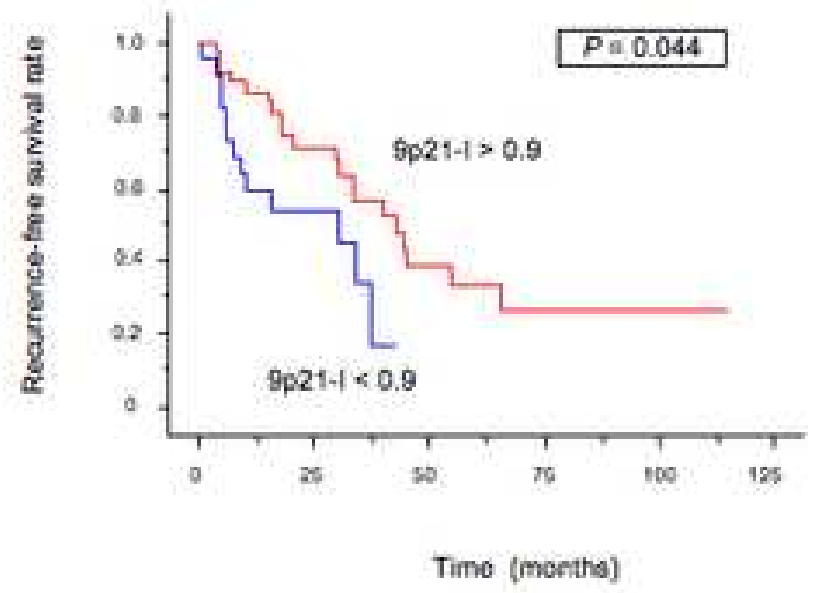


Table 1 Cytological grade and clinicopathologic features

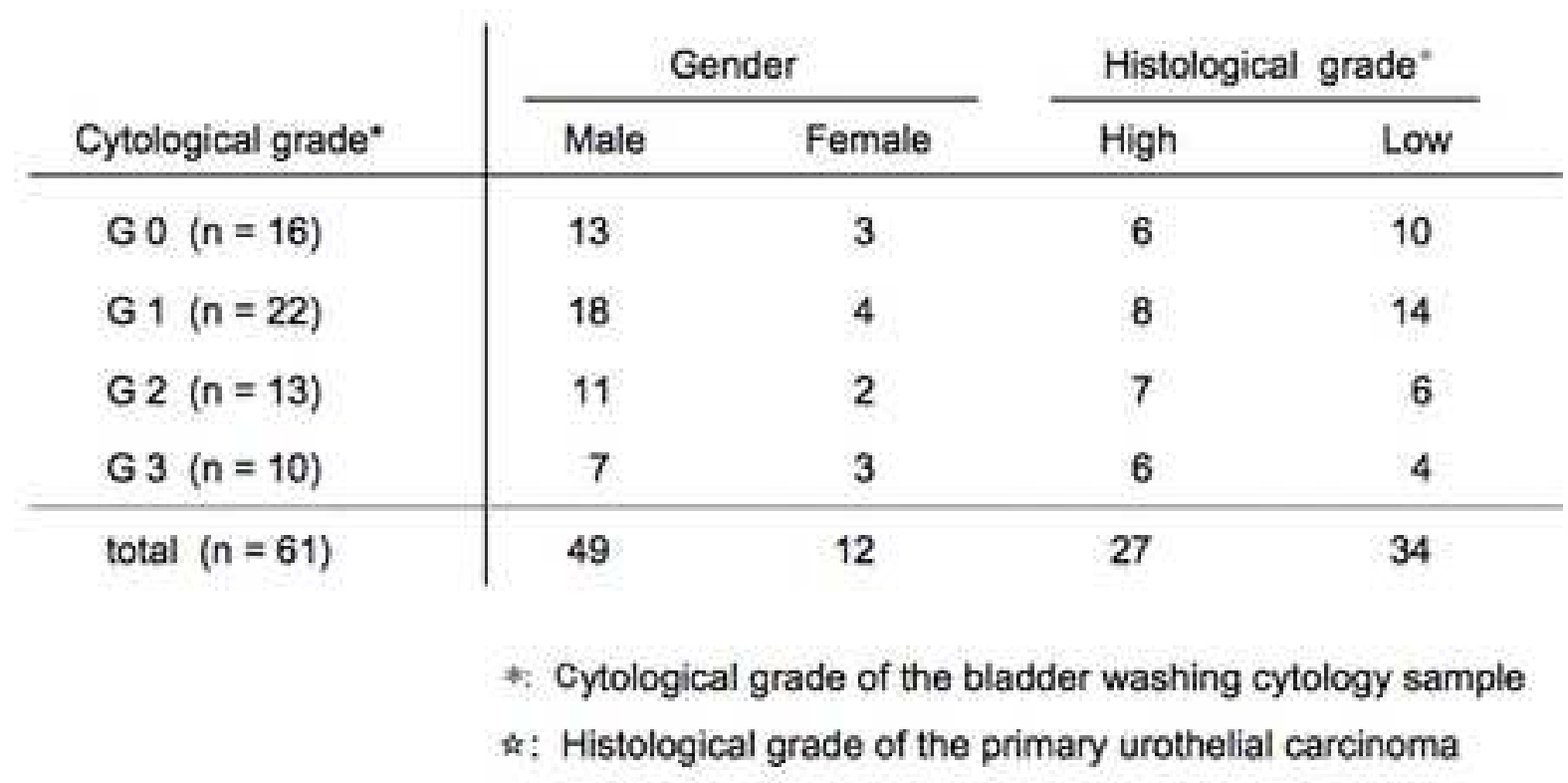


Table 2 Cytological grade and fluorescence in situ hybridization

\begin{tabular}{l|lll} 
Cytological grade & MN-9p21 (/nucleus) & MN-CH9 (/ nucleus) & $9 \mathrm{p} 21-1$ \\
\hline G0 $(n=16)$ & $2.09 \pm 0.191$ & $2.13 \pm 0.191$ & $0.984 \pm 0.018$ \\
G1 $(n=22)$ & $2.00 \pm 0.089$ & $2.14 \pm 0.161$ & $0.939 \pm 0.058$ \\
G2 $(n=13)$ & $1.84 \pm 0.146$ & $2.14 \pm 0.110$ & $0.859 \pm 0.066$ \\
G3 $(n=10)$ & $1.99 \pm 0.515$ & $2.51 \pm 0.605$ & $0.790 \pm 0.055$ \\
\hline total $(n=61)$ & $2.00 \pm 0.255$ & $2.20 \pm 0.321$ & $0.914 \pm 0.085$
\end{tabular}

Figures are described as mean \pm standard deviation.

MN-9p21: Mean 9p21 signal number per nucleus.

MN-CH9: Mean chromosome 9 centromere signal number per nucleus.

$9 p 21-1: 9 p 21$ index. $\quad 9 p 21-1$ is defined as MN-9p21/MN-CH9. 